\title{
Comparação entre hipertensão auto- referida e pressão arterial casual e presença de fatores de risco em funcionários da saúde de Bauru e Jaú
}

\author{
Comparision between self-reported hypertension and \\ casual blood pressure and presence of cardiovascular \\ risk factors among health workers of Bauru and Jau cities
}

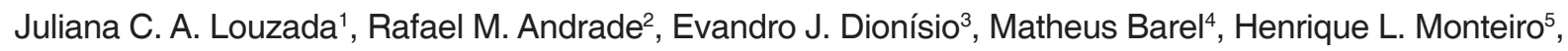
Sandra L. Do Amaral ${ }^{6}$

\begin{abstract}
RESUMO
Modelo: Estudo de prevalência. Objetivos: Avaliar a presença de hipertensão arterial auto-referida e comparar com os níveis de pressão arterial aferidos. Além disso, o trabalho se propôs a avaliar o grau de informação dos funcionários da saúde e determinar se o setor onde trabalha o funcionário influencia a Pressão Arterial (PA). Métodos: Foram avaliados 349 funcionários ( $46 \pm 10$ anos) das cidades de Bauru e Jaú, que responderam uma anamnese (dados pessoais, condição socioeconômica, presença de doenças, prática de exercícios físicos e setor ocupacional) e foram submetidos a uma avaliação da PA. Os avaliados se auto-referiram normotensos ou hipertensos. Valores de PA Sistólica $\geq 140 \mathrm{mmHg}$ e/ou PA diastólica $\geq 90 \mathrm{mmHg}$ foram considerados elevados. Dentre os avaliados, 198 foram submetidos à avaliações antropométricas e bioquímicas. Os resultados são apresentados como média \pm desvio padrão e distribuição de frequência. Utilizou-se teste "T-student" $(p<0,05)$. Resultados: Dentre os servidores, $16 \%$ se auto-referiram hipertensos, dos quais $56 \%$ apresentaram PA elevada e, destes, $91 \%$ utilizavam medicação antihipertensiva. Dentre os $84 \%$ que se auto-referiram normotensos, $24 \%$ apresentaram PA elevada e $8 \%$ tomavam medicamentos. A maioria dos funcionários de cada setor se autoreferiu normotenso, entretanto, grande parcela apresentou PA elevada, sendo esta mais frequente no setor "Saúde" (76,3\%). Conclusão: Os resultados sugerem que apesar da maioria dos funcionários se classificar como normotensos, uma parcela expressiva apresentou PA elevada e fazia uso de medicamentos inadequadamente, indicando que não tinham conhecimento do seu estado de saúde. Além disso, observou-se que o setor "Saúde" foi aquele que apresentou os maiores valores de PA.
\end{abstract}

Palavras-chave: Pressão Arterial. Fatores de Risco. Doenças Cardiovasculares. Profissionais da Saúde. Conhecimento/Detecção.

1 Educadora Física, UNESP/Bauru

2 Educador Físico - Especialista em Reabilitação Cardiovascular pelo Instituto Dante Pazzanese de Cardiologia

3 Aluno de Graduação do Curso de Licenciatura em Educação Física, UNESP/Bauru

4 Educador Físico. Especialista e Professor de Educação Física da Unidade de Reabilitação Cardíaca e Fisiologia do Exercício InCor/HC-FMUSP

5 Docente do Departamento de Educação Física da UNESP-Bauru 6 Docente do Departamento de Educação Física. Laboratório de Avaliação e Prescrição de Exercícios (LAPE). Departamento de Educação Física, Faculdade de Ciências -UNESP, BauruSP, Brasil.
Correspondência: Profa. Dra. Sandra Lia do Amaral UNESP - Faculdade de Ciências Departamento de Educação Física Av. Luiz Edmundo Carrijo Coube, 14-01 17033360 - Bauru/SP Fone/FAX: 55-14-31036082/55-14-31036071 slamaral@fc.unesp.br

Artigo recebido em 12/01/2010 Aprovado em 03/08/2010 


\section{Introdução}

A hipertensão arterial (HA) é uma doença multifatorial que, a cada ano, atinge maior parcela da população $\mathrm{O}^{1-2}$. É considerada um dos principais fatores de risco para o desenvolvimento de doenças cardiovasculares e sua prevalência é elevada em funcionários da saúde ${ }^{3}$. Nas cidades de Bauru e Jaú, a HA atinge cerca de $33 \%$ e $26 \%$ da população, respectivamente. Apesar das campanhas do governo no combate da HA, os custos médicos do tratamento são ainda elevados. ${ }^{4,5}$

As patologias relacionadas à condição ocupacional vêm ganhando maior atenção. A forte pressão psicológica, pouco tempo de lazer, turnos excessivos, baixos salários e dificuldades de acesso à assistência médica, associados ao estilo de vida sedentário, tornam os servidores da saúde potenciais candidatos a desenvolver problemas cardiovasculares. ${ }^{6,7,8}$

Apesar dos benefícios do exercício físico estarem em evidência, uma expressiva parcela da população é sedentária. No Brasil, 83\% não praticam exercícios habitualmente e, na região sudeste, esta proporção atinge 79,2\% dos adultos ${ }^{9}$. Além da presença de hipertensão e sedentarismo, outro grande problema que afeta a população é a desinformação com relação à saúde, o que prejudica o controle e o tratamento correto de diversas doenças ${ }^{10}$. Associado à falta de informações, acredita-se que somente $30 \%$ da população hipertensa é tratada adequadamente ${ }^{2}$, geralmente devido à falta de aderência ao tratamento. Os servidores da saúde são frequentemente acometidos por doenças crônicas como hipertensão, diabetes e obesidade, no entanto, não possuem conhecimento suficiente sobre os principais fatores de risco de doenças cardiovasculares $^{6-8}$. Portanto, o objetivo deste trabalho foi realizar uma análise descritiva da presença de hipertensão arterial auto-referida e compará-la com os valores de PA efetivamente aferidos. Além disso, o trabalho avaliou o grau de informação dos funcionários da saúde das cidades de Bauru e Jaú e determinou: 1) se o setor de trabalho influencia os valores de pressão arterial em um dia normal, bem como classe econômica e faixa etária; 2) se há presença de fatores de risco para doenças cardiovasculares nestes funcionários.

\section{Materiais e Métodos}

Foram avaliados 349 funcionários da área da saúde das cidades de Bauru e Jaú. A presente inves- tigação foi analisada e aprovada pelo Comitê de Ética da Secretaria Municipal de Saúde de Bauru. Todos os indivíduos leram e assinaram um Termo de Consentimento Livre e Esclarecido concordando com todos os procedimentos aos quais seriam submetidos durante $o$ protocolo de pesquisa.

As avaliações foram desenvolvidas com servidores pertencentes à Divisão Regional de Saúde-VI (DRS-VI), Bauru-Jaú e às Unidades Básicas de Saúde do Município de Bauru: Jardim Europa, Presidente Geisel, Vila Cardia, Centro, Vila Falcão, Vista Alegre, Godoy, Santa Edwirges, Redentor, Vila Dutra, BeijaFlor e Vila São Paulo, e ao SUS de Jaú. Os funcionários foram recrutados da seguinte forma: os da DRSVI receberam convite do diretor para participar de palestra sobre os fatores de risco cardiovasculares. Os servidores das unidades básicas de saúde foram convidados pelo avaliador, a partir de contato telefônico com o gestor da unidade, e as visitas ocorreram sempre num dia normal de trabalho. Em ambos os casos a participação foi voluntária.

Os funcionários que aceitaram participar da pesquisa responderam uma anamnese com dados pessoais, informações de condição socioeconômica, perguntas relacionadas à saúde como presença de doenças, cirurgias, medicamentos e tabagismo (foram considerados tabagistas os que fumavam pelo menos 1 cigarro por dia). Cada participante tinha de se referir como normotenso ou hipertenso de acordo com seu conhecimento em relação à patologia, bem como relatar sua rotina quanto à prática de exercícios físicos (foram considerados sedentários os que informaram não praticar exercícios físicos de forma regular pelo menos 2 vezes por semana) e setor ocupacional. Para determinar as características socioeconômicas foi utilizado o questionário baseado no critério de classificação econômica elaborado pelo IBGE ${ }^{11}$. Este questionário realiza um critério de classificação econômica que enfatiza o poder de compra das pessoas e famílias. Após o preenchimento da anamnese, os trabalhadores foram submetidos ao procedimento de aferição de pressão arterial (PA). Este procedimento foi realizado após 10 minutos de repouso, na posição sentada, pelo método auscultatório. Foi utilizado um esfigmomanômetro com aneróide (Solidor) calibrado e adequado à circunferência do braço, conforme preconizado pelas V Diretrizes Brasileiras de Hipertensão Arterial $^{2}$ e um estetoscópio (BD). Todos os avaliadores foram treinados e estavam aptos a aferir a PA seguindo as determinações das V Diretrizes Brasilei- 
ras de Hipertensão Arterial (2006). Foram considerados indivíduos com pressão elevada no dia da avaliação quando apresentaram a pressão arterial sistólica $(\mathrm{PAS}) \geq 140 \mathrm{mmHg}$ e/ou diastólica (PAD) $\geq 90 \mathrm{mmHg}$. Aqueles que estavam com a PAS $<140 \mathrm{mmHg}$ e a PAD $<90 \mathrm{mmHg}$ (incluídos os valores limítrofes de PA), foram classificados como pressão controlada. Os valores de PAM foram calculados a partir da fórmula: [(PAS-PAD)/3]+PAD.

Foram relacionados também os medicamentos anti-hipertensivos utilizados por cada servidor. Sete funcionários não autorizaram a publicação dos dados de PA.

Com o intuito de identificar se a posição ocupada dentro do Núcleo de Saúde poderia influenciar a PA, os funcionários foram subdivididos por setores de atuação: "Saúde" (médicos, enfermeiros, auxiliares de enfermagem e de dentista, nutricionista, farmacêuticos e psicólogos), "Administrativo" (cargos de direção e chefia, auxiliar e oficial administrativo), "Serviços gerais" (faxineiro, servente de limpeza, vigia, motorista, cozinheira) e "Outros" (assistente social, atendentes, agentes comunitários de saúde, auxiliar e técnico de laboratório, encarregado de setor).

Além da aferição da PA, parte dos avaliados (198 servidores pertencentes à Divisão Regional de Saúde-VI) foram submetidos à avaliações antropométricas e bioquímicas. Peso corporal (kg) e altura (m) foram utilizados para calcular o índice de massa corporal (IMC, $\left.\mathrm{kg} / \mathrm{m}^{2}\right)(<25$ bom, 25-30 limítrofe e $>30$ ruim $)^{12}$. A flexibilidade foi aferida por meio do teste de "sentar e alcançar" conforme protocolo descrito por Pollock and Wilmore ${ }^{11}$. Os valores de glicemia e perfil lipídico foram classificados segundo a I Diretriz da Sociedade Brasileira de Diabetes e IV Diretrizes em Dislipidemias, respectivamente ${ }^{13-14}$. Para efeito de análise, os valores limítrofes de cada variável foram agrupados com os elevados.

O risco cardiovascular destes 198 funcionários (43 homens e 155 mulheres) foi determinado a partir do protocolo proposto pela American Heart Association. ${ }^{15}$

As variáveis hemodinâmicas, bioquímicas e antropométricas foram apresentadas sob a forma de média \pm desvio padrão da média. Os dados descritivos foram organizados sob a forma de distribuição de frequência (\%) conforme suas respostas na anamnese e valores aferidos de PA. Para análise dos valores pressóricos por faixa etária e sexo, utilizou-se o teste "T-student" $(\mathrm{p}<0,05)$.

\section{Resultados}

Dos 349 indivíduos avaliados, 269 eram da cidade de Bauru e 80 de Jaú, dos quais 57 do sexo masculino (16\%) e 292 do feminino (84\%), com média de idade de $46 \pm 10$ anos. Dentre estes funcionários, $37,1 \%$ tinham até 39 anos, sendo $30,5 \%$ do sexo feminino e $6,6 \%$ do sexo masculino. A faixa etária de 40-49 anos foi representada por $33 \%$ dos funcionários $(28,9 \%$ mulheres e $4,1 \%$ homens). Dentre os avaliados com idade entre 50-59 anos, encontramos $24,4 \%$ dos indivíduos, dos quais $19,8 \%$ eram mulheres e $4,6 \%$ homens e $5,7 \%$ das pessoas tinham idade superior a 60 anos (4,6\% do sexo feminino e $1,1 \%$ do sexo masculino).

A Figura 1 ilustra as informações auto-referidas dos indivíduos e os valores pressóricos encontrados no dia da avaliação. Do total de avaliados, $16 \%$ (57 pessoas) se auto-referiram hipertensos e 84\% (292 pessoas) normotensos. Dentre os auto-referidos hipertensos, $44 \%$ ( 25 pessoas) apresentaram a PA controlada com o uso regular de medicamentos antihipertensivos. Por outro lado, 56\% (32 pessoas) estavam com a PA elevada no dia da avaliação, dos quais 91\% (29 pessoas) faziam uso de medicamentos antihipertensivos. No que diz respeito aos trabalhadores que se auto-referiram normotensos, observou-se que $76 \%$ (222 pessoas) estavam com a PA controlada, dos quais $2 \%$ (5 pessoas) utilizavam fármacos antihipertensivos. Vinte e quatro por cento (70 pessoas) apresentaram PA elevada no momento da avaliação, desses, $8 \%$ (6 pessoas) faziam uso regular de medicamentos anti-hipertensivos.

Na divisão dos funcionários por ocupação (Figura 2) observou-se a proporção de $27 \%$ na área da "Saúde", 20\% setor "Administrativo", 11\% "Serviços gerais" e 33\% "Outros" (9\% não informaram). Os resultados referentes à classificação auto-referida foram relacionados com os setores ocupacionais. A figura $2 \mathrm{~A}$ ilustra que nos quatro setores avaliados, a maioria dos funcionários se auto-referiram normotensos. Esta condição auto-referida estava presente em $89 \%$ dos funcionários da "Saúde", $80 \%$ do setor "Administrativo", $93 \%$ do setor de "Serviços gerais" e 79\% do setor "Outros". Porém, quando os valores de PA foram aferidos, observou-se um contraste, ou seja, no setor da "Saúde", 76\% dos funcionários apresentaram PA elevada, no setor "Administrativo" 65\%, no setor de "Serviços gerais" $55 \%$ e no setor "Outros 74\% (Figura 2B). 


\section{TOTAL DE PESSOAS $=349$}

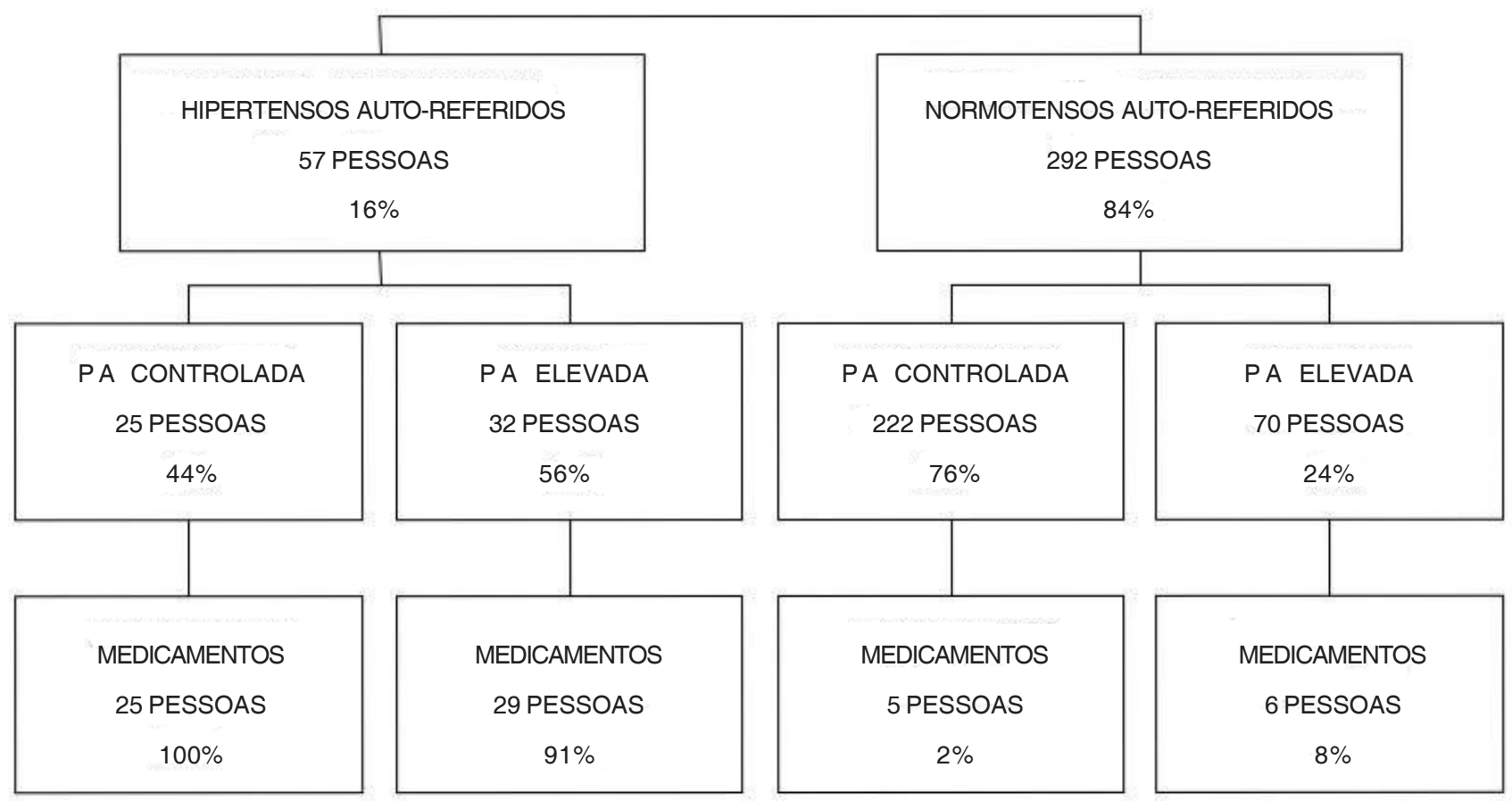

Figura 1: Fluxograma com distribuição de frequência dos funcionários da saúde de acordo com informação auto-referida, valores de pressão mensurados na avaliação e tratamento medicamentoso.

Figura 2 (A)

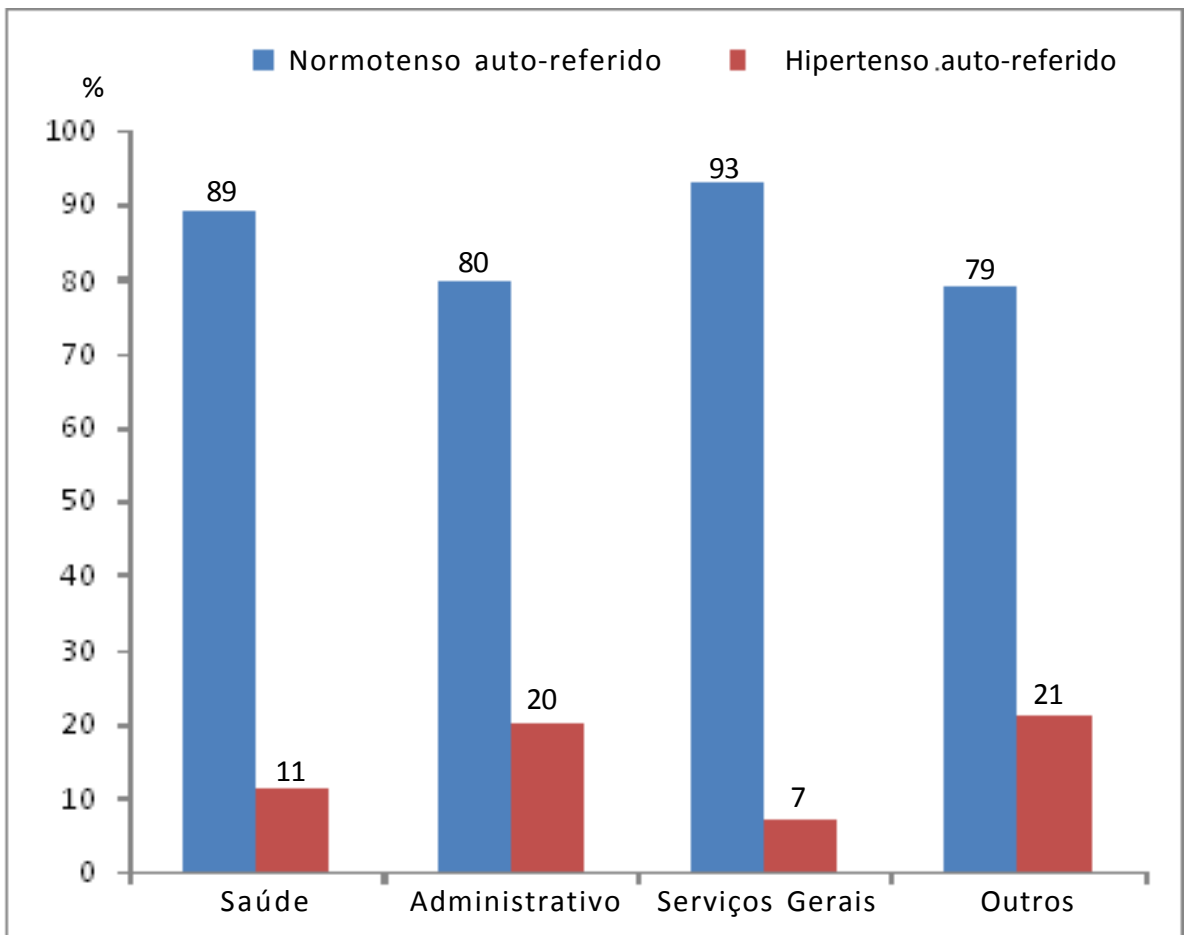

Figura 2 (A): Distribuição de frequência dos funcionários da saúde de acordo com informação auto-referida, por ocupação. 


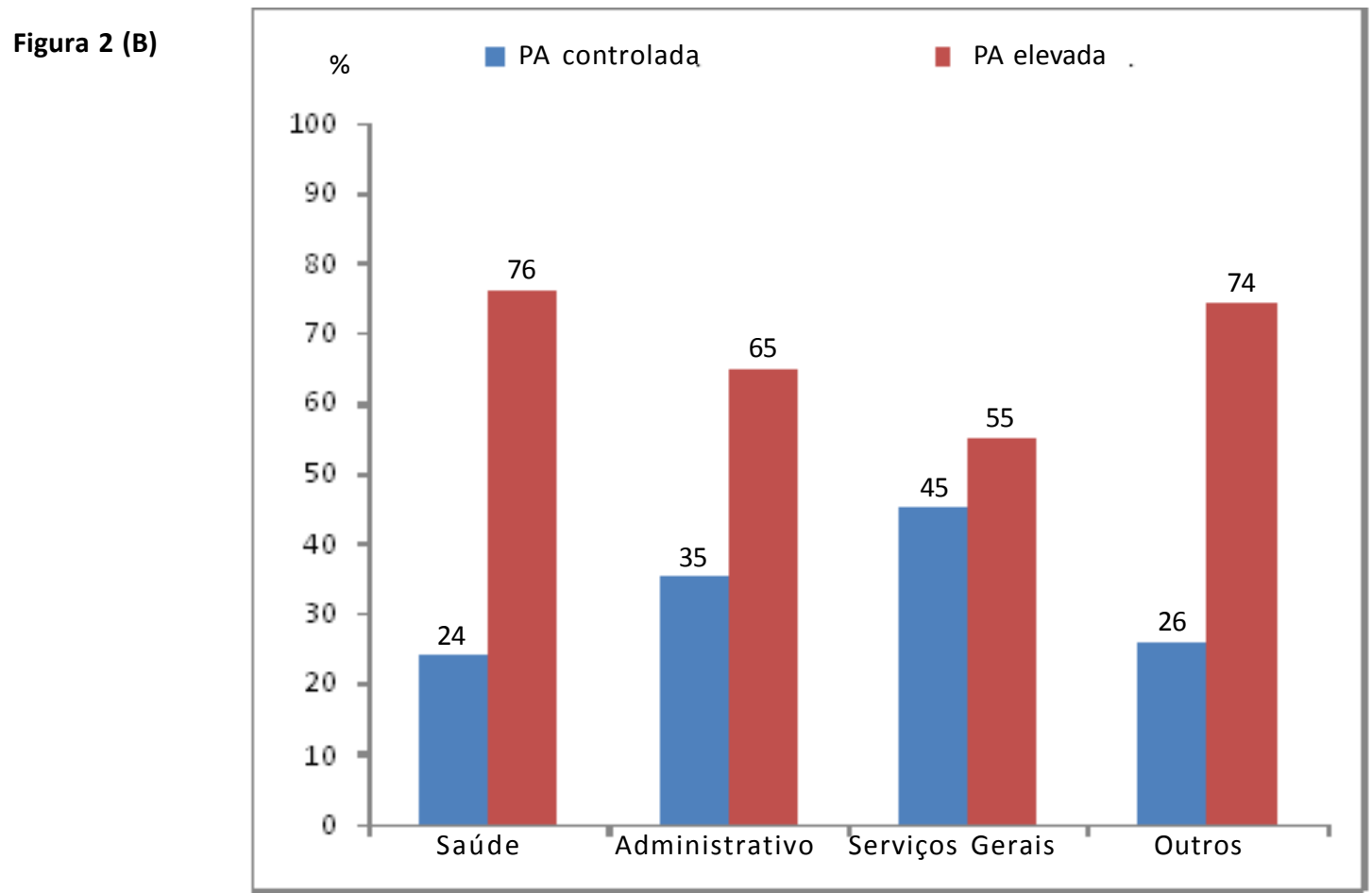

Figura 2 (B): Distribuição de frequência dos funcionários da saúde de acordo com os valores pressóricos mensurados na avaliação, por ocupação.

As classes sociais predominantes dentre os avaliados foram B e C (44\% e $41 \%$, respectivamente), seguidas das classes D, A e E $(8 \%, 3 \%$, e $1 \%$, respectivamente). Três por cento das pessoas não informaram. A informação auto-referida, bem como os valores pressóricos obtidos no dia da avaliação, foram relacionados com a condição socioeconômica dos funcionários. A hipertensão auto-referida estava presente em $20 \%$ dos funcionários da classe social A, $19 \%$ da B, $10 \%$ da C, $25 \%$ da D e $50 \%$ da E. Dentre os auto-referidos normotensos, as classes sociais que apresentaram o maior número de funcionários foram as B e C ( $81 \%$ e $90 \%$, respectivamente). A Figura 3 ilustra a média dos valores pressóricos de pressão arterial média (PAM) observados no dia da avaliação estratificados por faixa etária (até 39 anos, entre 40 e 49 anos, entre 50 e 59 anos e acima de 60 anos) e comparados por sexo. Pode-se observar que somente na faixa etária até 39 anos, as mulheres apresentaram valores de PAM significativamente menores que os homens $(p<0,05)$. O mesmo foi observado para PAS e PAD (dados não demonstrados). A quantidade de exercícios praticada por cada funcionário não se correlacionou com os valores de PA (dados não demonstrados).Além dos valores de PA, foram avaliados outros fatores de risco para doenças cardiovasculares em parte dos funcionários (198 pessoas). A Figura 4 ilustra a porcentagem de pessoas que apresentavam valores limítrofes e elevados das variáveis analisadas. O principal fator de risco apresentado pelos funcionários da saúde foi o nível de lipoproteína de alta densidade (HDL-C) reduzido, encontrado em 75\% dos funcionários. O risco coronariano, caracterizado por histórico familiar de doenças cardiovasculares e associação de outros fatores de risco, foi o segundo fator encontrado, totalizando $74 \%$ dos avaliados. Em terceiro lugar, acometendo $56 \%$ dos funcionários, pôdese observar o sedentarismo. Mais da metade dos servidores estavam acima do peso ideal (54\%). Valores de colesterol total acima da normalidade foram encontrados em $49 \%$ dos servidores, lipoproteína de baixa densidade (LDL-C) em 41\%, tabagismo em 33\%, triglicerídeos em $24 \%$ e glicemia de jejum elevada em $10 \%$ dos funcionários. A Tabela 1 ilustra a média e desvio padrão das variáveis antropométricas, bioquímicas e hemodinâmicas estudadas. 


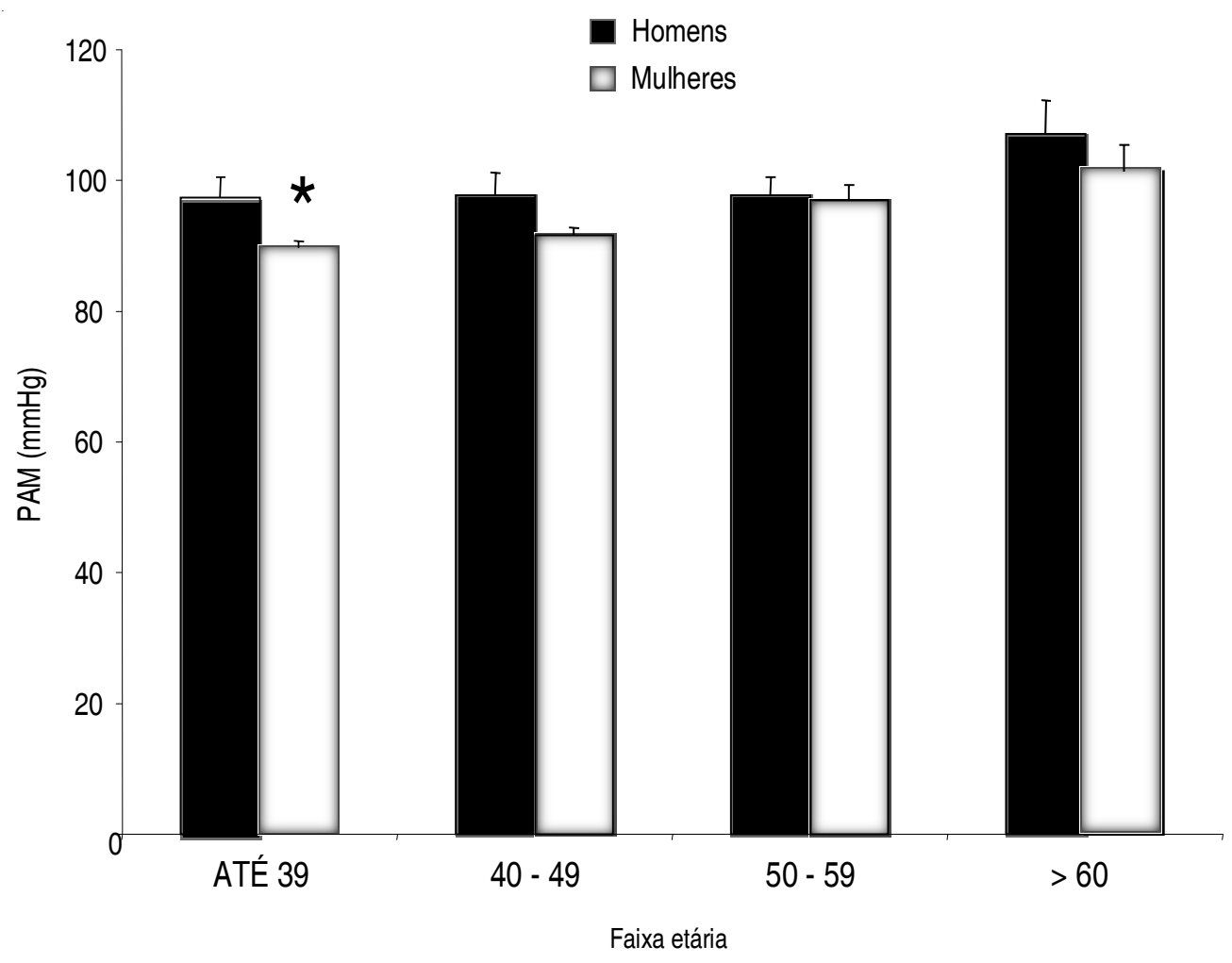

Figura 3: Comparação dos valores de pressão arterial média por sexo, segundo faixa etária. Foram avaliados 57 homens e 292 mulheres. Significância: * vs homens, $p<0,05$.

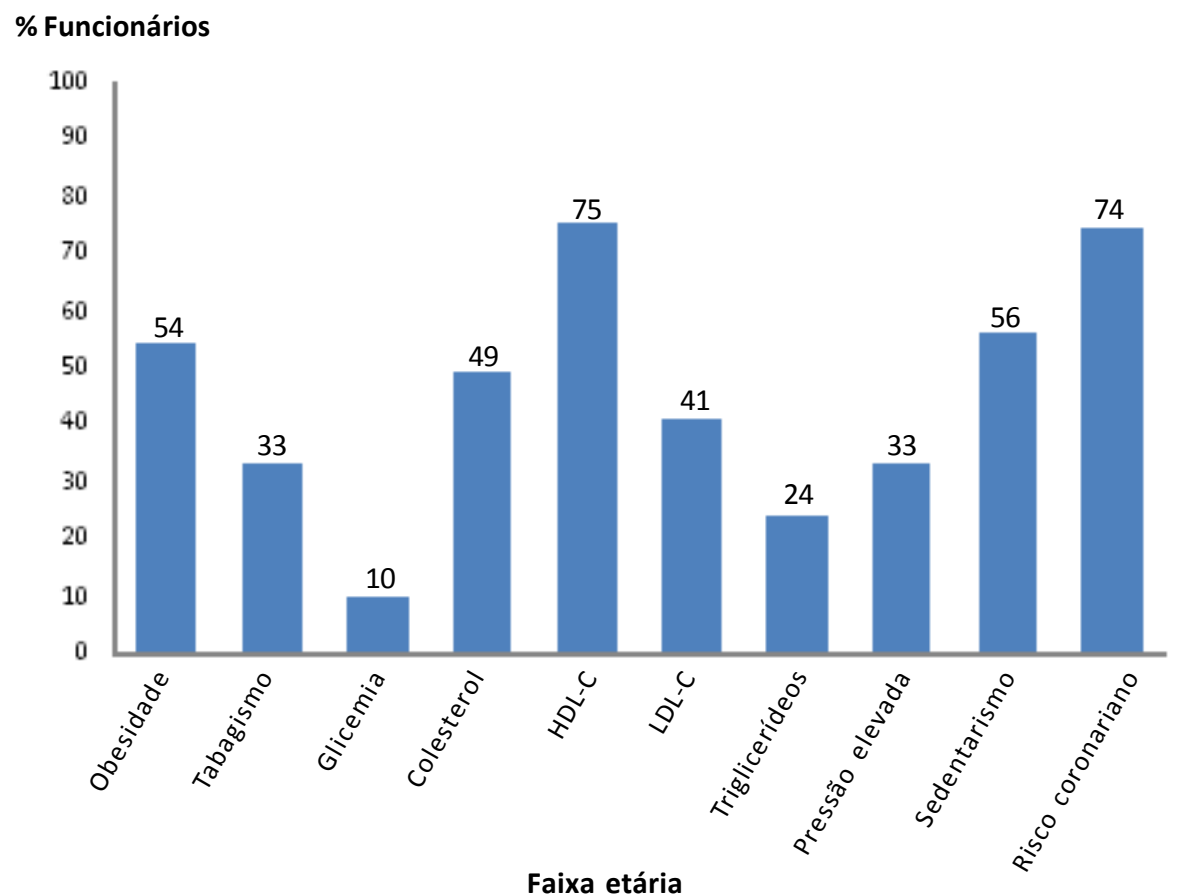

Figura 4: Distribuição da frequência dos fatores de risco dos funcionários da saúde. HDL-C: lipoproteína de alta densidade; LDL-C: lipoproteína de baixa densidade (valores elevados agrupados com os limítrofes, Diretriz da Sociedade Brasileira de Diabetes, 2007 e IV Diretriz Brasileira de Dislipedimas, 2007). 


\section{Tabela 1}

Variáveis antropométricas, bioquímicas e hemodinâmicas dos funcionários avaliados

\begin{tabular}{lcr}
\hline Variáveis & Homens $(n=43)$ & Mulheres $(n=155)$ \\
\hline Peso $(\mathrm{kg})$ & $79,7 \pm 15,2$ & $67,8 \pm 14,4$ \\
Altura $(\mathrm{cm})$ & $138 \pm 28$ & $158 \pm 6,5$ \\
IMC $\left(\mathrm{kg} / \mathrm{cm}^{2}\right)$ & $27 \pm 5$ & $27 \pm 5$ \\
Flexibilidade $(\mathrm{cm})$ & $17 \pm 7$ & $23 \pm 9$ \\
Glicemia $(\mathrm{mg} / \mathrm{dL})$ & $96 \pm 28$ & $93 \pm 9$ \\
Colesterol total $(\mathrm{mg} / \mathrm{dL})$ & $214 \pm 51$ & $205 \pm 38$ \\
HDL-colesterol (mg/dL) & $43 \pm 12$ & $52 \pm 12$ \\
LDL-colesterol (mg/dL) & $145 \pm 69$ & $129 \pm 34$ \\
Triglicérides (mg/dL) & $213 \pm 239$ & $117 \pm 66$ \\
Pressão arterial sistólica (mmHg) & $127 \pm 17$ & $120 \pm 23$ \\
Pressão Arterial diastólica $(\mathrm{mmHg})$ & $82 \pm 13$ & $78 \pm 13$ \\
\hline
\end{tabular}

HDL - lipoproteína de alta densidade; LDL - lipoproteína de baixa densidade; IMC - Índice de massa corporal.

\section{Discussão}

Um dos principais achados deste estudo foi a alta presença de PA elevada (29\%) associada à desinformação sobre a condição de saúde dos funcionários da área. Pôde-se observar que o setor denominado "saúde" constitui o grupo que apresentou a maior taxa de PA elevada, embora a maioria desses indivíduos se auto-referiram como normotensos.

A partir dos resultados da presente pesquisa, pôde-se constatar que a maioria dos servidores avali$\operatorname{ados}(84 \%)$ se auto-referiram normotensos e apenas $16 \%$ se consideraram hipertensos, no entanto a PA estava elevada em 29\% dos casos. Mesmo dentre os auto-referidos normotensos, 24\% apresentaram níveis elevados de PA no dia da avaliação. Esta discrepância de resultados entre informação auto-referida e valores aferidos pode refletir um problema presente nos dias de hoje, que é a falta de informação quanto à condição de saúde. ${ }^{10,16,17}$ A esse respeito, Peres et $\mathrm{al}^{10}$ também constataram que, dentre os pacientes de uma unidade básica de saúde na cidade de Ribeirão Preto, $38 \%$ não tinham conhecimento sobre a doença e, dentre aqueles que associaram HA com pressão alta, quase $50 \%$ não sabiam definir o que era PA elevada. A comparação entre o estudo de Peres et $\mathrm{al}^{10} \mathrm{e}$ a presente pesquisa sugere que os servidores são mais informados que os pacientes.

A hipótese deste trabalho, ao realizar esta investigação com profissionais da saúde, era de que o grau de informação sobre a hipertensão auto-referida fosse maior. No entanto, ao contrário do esperado e, em consonância com os achados de Mion et al, ${ }^{16} 24 \%$ dos funcionários auto-referidos normotensos e que apresentavam a PA elevada, $8 \%$ faziam uso de medicação anti-hipertensiva, o que destaca ainda mais a condição de desinformação destes trabalhadores. No estudo de Mion et al ${ }^{16}, 51 \%$ dos funcionários de um hospital da cidade de São Paulo, que se auto-referiram hipertensos, estavam com a PA elevada e, dentre os $38 \%$ que apresentaram PA elevada, $91 \%$ se autoreferiram normotensos.

Outro problema observado nos funcionários estudados foi a ineficiência do tratamento anti-hipertensivo. Entre os auto-referidos hipertensos, somente $44 \%$ apresentavam PA controlada por medicamentos, entretanto, $56 \%$ estavam com a PA elevada, mesmo com o uso dos fármacos. Este comportamento pode ser explicado por diversas razões, entre as quais insônia, preocupações 2,6,16, síndrome do "jaleco branco" ${ }^{2,19}$ ou ineficiência do tratamento. ${ }^{2,20,21}$ De fato, tem-se demonstrado que apenas pequena parcela da 
população adere ao tratamento medicamentoso ${ }^{20} \mathrm{e}$ mesmo entre os aderentes, somente $30 \%$ dos casos são efetivamente controlados. ${ }^{22}$

A presente investigação apontou presença de $29 \%$ de pessoas com PA elevada (apesar dos autoreferidos hipertensos serem apenas $16 \%$ ), número semelhante às porcentagens de pacientes hipertensos encontradas em Bauru e Jaú (33\% e 26\%, respectivamente) conforme DATASUS. ${ }^{23}$

Outros estudos semelhantes realizados em diversas regiões do Brasil apontam que a prevalência de hipertensão é alta, variando entre $12 \%$ a $46 \%$ 16, 21 , ${ }^{24-29}$, dados estes que estão de acordo e até superiores aos resultados encontrados nesta pesquisa. Os trabalhos acima citados indicam também que a incidência de HA vem aumentando gradativamente com o decorrer dos anos, particularmente nas duas últimas décadas. Estas pesquisas foram realizadas com populações em geral e quase nada se sabe sobre a prevalência de HA especificamente nos funcionários da saúde. Mion et al ${ }^{16}$ observaram taxa de prevalência de 26\% nos funcionários da saúde do hospital das clínicas de São Paulo, resultado semelhante aos $29 \%$ encontrados na presente investigação.

A literatura tem apontado que o tipo de trabalho pode influenciar diretamente na qualidade de vida e de saúde do empregado ${ }^{8}$. Por esta razão, os servidores foram agrupados em setores e, os valores de PA considerados de acordo com cada grupo. Os resultados da presente pesquisa revelaram um fato interessante: em todos os setores, a maioria dos avaliados se autoreferiram normotensos, com porcentagem acima de $75 \%$. No entanto, quando os valores de PA foram aferidos, houve prevalência de PA elevada em todas as áreas ocupacionais. A área que parece ter maior influência sobre os níveis pressóricos foi a "saúde", onde estão agrupados médicos, enfermeiros, auxiliares de enfermagem, dentistas, nutricionistas, farmacêuticos e psicólogos. O setor "outros", que compreende assistentes sociais, atendentes, agentes comunitários de saúde, auxiliares/técnicos de laboratório e encarregados de setor, apresentou a segunda maior taxa de PA elevada, ficando o setor "administrativo" e "serviços gerais" em $3^{\circ}$ e $4^{\circ}$ lugares, respectivamente. Divergindo desses resultados, Mion et $\mathrm{al}^{16}$ demonstraram que a área que mais apresentava PA alterada foi aquela em que médicos não estavam presentes. No entanto, esta discrepância pode ser justificada pelas diferentes composições dos grupos. Para o presente estudo, as pessoas do setor administrativo foram separadas dos auxiliares de serviço, encarregados de setor, cozinheiros, serventes, entre outros, enquanto no estudo de Mion et $\mathrm{al}^{16}$ foram agrupados.

Apesar de alguns estudos que correlacionam o nível socioeconômico com valores de PA demonstrarem que pessoas pertencentes à classes sociais inferiores apresentam valores de PA superiores ${ }^{16,21,30,31,32}$, o presente estudo não confirmou esta associação. No setor da saúde, que continha $76 \%$ de hipertensos, a classe predominante é a B e no setor "outros", com 73\% de hipertensos, há predomínio da classe C. O setor de serviços gerais, que agrupa pessoas das classes sociais B, C e D apresentou somente $55 \%$ de pessoas com PA elevada. A condição socioeconômica também parece não influenciar o grau de conhecimento sobre a hipertensão ou condição de saúde, ao contrário do atestado por outros pesquisadores. ${ }^{16,21,30,31,32}$ Os resultados sugerem que os indivíduos pertencentes às classes sociais $\mathrm{A} \mathrm{e} \mathrm{C}$ tinham mais conhecimento de seu estado de saúde quando as informações autoreferidas foram correlacionadas com os valores de PA, principalmente os normotensos. Contudo, o fato de haver correlação positiva entre informação autoreferida e valores de PA pode indicar também, a falta de conhecimento e a ineficiência do tratamento antihipertensivo, pois os auto-referidos hipertensos e tratados farmacologicamente deveriam estar com a PA controlada. Nesta direção, é possível sugerir que os funcionários pertencentes às classes $\mathrm{B}$ e $\mathrm{D} / \mathrm{E}$ apresentam mais conhecimento sobre o assunto, bem como aderência ao tratamento.

A tendência da PA ser maior nos homens e aumentar com a idade foi confirmada neste estudo. Até os 39 anos, os homens apresentaram valores de PAS, PAM e PAD superiores quando comparados com as mulheres de mesma faixa etária. Esta prevalência de PA elevada nos homens se faz presente em quase todos os estudos que consideram o gênero como fator que influencia a PA..$^{2,9,29,33}$

Apesar da eficiência do exercício físico em controlar a PA ser amplamente reconhecida, a taxa de sedentarismo ainda é muito elevada, tanto no Brasil quanto no mundo. ${ }^{9,24,25,28,29}$ Dados do presente estudo revelaram que em Bauru e Jaú estas prevalências atingem $68 \%$ e $60 \%$, respectivamente. Quando reunidos, os valores apontam que $63 \%$ dos funcionários da saúde eram sedentários, taxa superior àquelas encontradas por Dioguardi et $\mathrm{al}^{34}$. Apenas um estudo na literatura apontou porcentagem reduzida de sedentá$\operatorname{rios}^{35}$, no entanto, o nível de atividade física foi avalia- 
do pelo IPAQ-8, que tem sido questionado quanto a sua fidedignidade em estabelecer o grau de atividade física. Outras pesquisas têm demonstrado correlação inversa entre níveis de atividade física e hipertensão $0^{36-}$ ${ }^{37}$, entretanto, esta correlação não foi observada no presente trabalho. Vários fatores podem ter contribuído para esta resposta negativa: nos questionários aplicados, os funcionários deveriam responder se praticavam atividades físicas ou não. No entanto, se existe desinformação com relação à condição de saúde, o conceito de exercício físico também pode não estar claro para estas pessoas. Muitos consideram praticar esportes uma vez por semana como exercício e por isso se auto-classificaram ativos. Além disso, não havia, no questionário aplicado, uma questão específica sobre o tempo dispendido no exercício físico e gasto energético. Por esse motivo, é plausível a hipótese de que o número de ativos não seja condizente com a realidade. Pôde-se observar que havia grande número de pessoas auto-referidas ativas com a PA descontrolada e, sedentárias com a PA normal. Este fato contribui para uma série de questionamentos com relação à forma de se avaliar a condição física por meio de questionários. ${ }^{38,39,40}$

Associado à HA e sedentarismo, outros fatores de risco têm contribuído de forma significativa para aumentar a mortalidade por doenças cardiovasculares. Apesar de não ser objetivo específico deste tra- balho, parte dos funcionários da saúde foi submetida à avaliações complementares com o intuito de se verificar a presença de fatores de risco. Neste sentido, acredita-se que os funcionários da saúde são pouco assistidos e apresentam grande número de fatores de risco associados, o que contribui para o desenvolvimento de doenças cardiovasculares como demonstrado por inúmeros outros estudos. ${ }^{24,25,35,41}$

Os resultados sugerem que apesar da maioria dos funcionários se classificar como normotensos, uma parcela expressiva apresentou PA elevada e fazia uso de medicamentos inadequadamente, indicando que não tinham conhecimento do seu estado de saúde. Além disso, observou-se que o setor "Saúde" foi aquele que apresentou os maiores valores de PA dos funcionários.

\section{Agradecimentos}

Financiamento: Pró-reitoria de Extensão da UNESP/PROEX.

Os autores agradecem a: i) Diretoria da Divisão Regional de Saúde, DRS-VI de Bauru, em especial Sr. Amilton Alves Teixeira e Vera Helena Perlatti D’Alpino do NGA-25 de Jaú pela contribuição no desenvolvimento deste estudo, pelo incentivo dado a seus servidores e pela realização dos exames laboratoriais; ii) aos monitores do LAPE (Laboratório de Avaliação e Prescrição de Exercícios) que auxiliaram nas avaliações e análises de dados.

\begin{abstract}
Model: Prevalence study. Objectives: To evaluate the presence of self-reported hypertension to compare with blood pressure measurements. Besides, this work investigated health information level of workers and if the job position has any influence on blood pressure (BP).

Methods: This study evaluated 349 health workers $(44 \pm 10$ years old) from Bauru and Jau cities, who answered some questions about history of health condition, use of medicines, past surgeries as well as social, scholar and physical conditions and had their blood pressure measured. Each subject selfreported as normotensive or hypertensive. Values of systolic $\geq 140 \mathrm{mmHg}$ and/or diastolic PA $\geq 90$ $\mathrm{mmHg}$ were considered elevated. Among the health workers evaluated, 198 were submitted to anthropometric and biochemical evaluations. Values are presented as means \pm SD and frequency of distribution. It was used T-student test $(\mathrm{p}<0.05)$. Results: From all workers evaluated only $16 \%$ self-reported as hypertensive, which $56 \%$ presented high BP, however $91 \%$ used to take antihypertensive medicines. Among the $84 \%$ who self-reported as normotensive, $24 \%$ presented high BP and $8 \%$ used to take medicines. Although most of the employees of each section self-reported as normotensive, more than a half presented high BP and which was more common in the health's section (76.3\%). Conclusion: These results suggest that besides the majority of the employees self-reported as normotensive, an elevated number of health workers presented high blood pressure and used to take medicines inappropriately, which indicates that they did not have enough knowledge about their health. Furthermore, it was observed that Health Section presented the higher blood pressure values.
\end{abstract}

Keywords: Blood Pressure. Risk Factors. Cardiovascular Diseases. Health Personnel. knowledge/ Detection. 


\section{Referências}

1. Chobanian AV, Bakris GL, Black Hr, Cushman WC, Green LA, Izzo JLJ, et al and Joint National Committee on Prevention, Detection, Evaluation, and Treatment of High Blood Pressure. National Heart, Lung, and Blood Institute; National High Blood Pressure Education Program Coordinating Committee. Seventh report of the Joint National Committee on Prevention, Detection, Evaluation, and Treatment of High Blood Pressure. Hypertension. 2003; 42:1206-52.

2. V Diretrizes Brasileiras de Hipertensão. Rev Bras Hipertens.2006; (4):256-312.

3. Mokdad AH, Marks JS, Stroup DF, Gerberdng JL. Actual causes of death in United States. J Am Med Assoc. 2004; 291: 1238-45.

4. Stafilas PC, Sarafadis PA, Lasaridis AN, Aletras VH, Niakas DA. An Economic Evaluation of the 2003. European Society of Hypertension-European Society of Cardiology Guidelines for the Management of Mild-to-Moderate Hypertension in Greece. Am J Hypertens. 2005; 9:1233-40.

5. Rolim LM, Amaral SL, Monteiro HL.Hipertensão e Exercício: custos do tratamento ambulatorial, antes e após a adoção da prática regular e orientada de condicionamento físico. Hipertensão 2007; 10: 54-61

6. Matthews KA, Katholi RC, McCreath H, Whooley MA, Williams DR, Zhu S, et al. Blood Pressure Reactivity to Psychological Stress Predicts Hypertension in the CARDIA Study. Circulation.2004; 110:1-5.

7. Hwang LC, Tsai CH, Chen TH. Overweight and obesity-related metabolic disorders in hospital employees. J Formos Med Assoc 2006; 105:56-63.

8. Couto HA, Vieira FLH, Lima EG. Estresse ocupacional e hipertensão arterial sistêmica. Rev Bras Hipertensão 2007; 2:112-5.

9. Fatores de risco. Atlas - corações do Brasil. Sociedade Brasileira de Cardiologia 2005; 1: 40-54.

10. Péres DS, Magna JM, Viana LA. Portador de hipertensão arterial: atitudes, crenças, percepções, pensamentos e práticas. Rev Saúde Pública 2003; 5: 635-42.

11. Pereira MG. Epidemiologia Moderna: teoria e prática. Rio de Janeiro: Guanabara Koogan, 1996.

12. Pollock ML \& Wilmore. Exercícios na saúde e na doença: avaliação e prescrição para a prevenção e reabilitação 1993; Medsi, p.736.

13. Diretrizes da Sociedade Brasileira de Diabetes, Tratamento e acompanhamento do diabetes mellitus. Sociedade Brasileira de Diabetes, Rio de Janeiro, 2007; 14

14. IV Diretrizes em Dislipidemia. Sociedade Brasileira de Cardiologia, Rio de Janeiro, 2007; 10.

15. Kavey REW, Daniels SR, Lauer RM, Atkins DL, Hayman LL, Taubert K. American heart association guidelines for primary prevention of atherosclerotic cardiovascular disease beginning in childhood. Circulation. 2003; 107:1562-6.

16. Mion Jr D, Pierin AMG, Bambirra AP, Assunção JH, Monteiro $\mathrm{JM}$, Chinen RY, et al. Hypertension in employees of a university general hospital. Rev Hosp Clin Fac Med S Paulo 2004; 6:329-36
17. Guimarães MV, Ribas LFO. Avaliação da compreensão dos pacientes hipertensos a respeito da hipertensão arterial e seu tratamento versus controle pressórico. Rev Bras Med Fam comunidade. 2006; 1:152-64

18. Gangwisch JE, Heymsfield SB, Boden-Albala B, Buijs RM, Kreier F, Pickering TG, et al. Short Sleep Duration as a Risk Factor for Hypertension analyses of the First National Health and Nutrition Examination Survey. Hypertension. 2006; 47: 833-9.

19. Corrêa TD, Namura JJ, Silva CAP, Castro MG, Meneghini A, Ferreira C. Systemic hypertension: latest information on its epidemiology, diagnosis and treatment. Arq Med ABC 2005; 2:91-101

20. Strelec AMG, Pierin AMG, Mion Jr D. A influência do conhecimento sobre a doença e atitude frente à tomada dos remédios no controle da hipertensão arterial. Arq Bras Cardiol. 2003; 81: 349-54.

21. Gus I, Harzheim E, Zaslavsky, Medina C; Gus M. Prevalence, awareness and control of systemic arterial hypertension in the state Rio Grande do Sul. Arq Bras Cardiol. 2004; 83:429 33.

22. Coelho EB, Neto MM, Palhares R, Cardoso MCM, Geleilete TJM, Nobre F. Relação entre assiduidade às consultas ambulatoriais e o controle da pressão arterial em pacientes hipertensos. Arq Bras Cardiol. 2005; 3:157-61.

23. Datasus. Ministério da Saúde. Disponível em: ttp:// w3.datasus.gov.br/datasus/datasus.php.

24. Forjaz CLM, Tinucci T, Bartholomeu T, Fernandes TEM, Casagrande V, Massucato JG. Assessment of the cardiovascular risk and physical activity of individuals exercising at a public park in the city of São Paulo. Arq Bras Cardiol. 2002; 1:43-50

25. Gus I, Fischmann A, Medina C. Prevalence of risk factors for the arterial coronary disease in the Brazilian state of Rio Grande do Sul. Arq Bras Cardiol. 2002; 78:478-83.

26. Lima-Costa MF, Peixoto SV, Firmo JOA. Validade da hipertensão auto-referida e seus determinantes (projeto Bambuí). Rev Saúde Pública 2004; 5:637-42.

27. Rosini N, Machado MJ, Xavier HT. Study of the prevalence and multiplicity of cardiovascular risk factors in hypertensive individuals from the city of Brusque, SC, Brazil. Arq Bras Cardiol. 2006; 86:219-222.

28. Zaitune MPA, Barros MBA, César CLG, Carandina L, Goldbaum $M$. Hipertensão arterial em idosos:prevalência, fatores associados e práticas de controle no Município de Campinas São Paulo, Brasil. Cad Saúde Pública 2006; 2:285-94.

29. Jardim PCBV, Gondim MRP, Monego ET, Moreira HG, Vitorino PVO, Souza WKSB, et al. Hipertensão Arterial e alguns fatores de risco em uma capital brasileira. Arq Bras Cardiol. 2007; 4:452-7.

30. Ezeamama AE, Viali S, Tuitele J, McGarvey ST. The influence of socioeconomic factors on cardiovascular disease risk factors in the context of economic development in the Samon archipelago. Soc Sci Med. 2006; 63:2533-45.

31. Godoy MF, Lucena JM, Miquelin AR, Paiva FF, Oliveira DLQ, Augustin Jr JL, et al. Cardiovascular Mortality and its Relation to Socioeconomic Levels among Inhabitants of São José do Rio Preto, São Paulo State, Brazil. Arq Bras Cardiol. 2007; 2:176-82 
32. Grotto I, Huerta M, Grossman E, Sharabi Y. Relative impact of socioeconomic status on Blood Pressure: lessons from a Large-Scale Survey of young adults. Am J Hypertens. 2007; 20:1140-5.

33. Stranges S, Wu T, Dorn JM, Freudenheim JL, Muti P, Farinaro $\mathrm{E}$, et al. Relationship of alcohol drinking pattern to risk of hypertension: a population-based study.Hypertension. 2004; 44:813-9.

34. Dioguardi GS, Ghurayeb N, Reginato LE, Baptista C, Savioli F, Camargo CL Atividade física em médicos. Rev Soc Cardiol Estado de São Paulo 1996; 1:117-20.

35. Viebig RF, Valero MP, Araújo F, Yamada AT, Mansur AJ. Cardiovascular health profile of adult population from the metropolitan region of São Paulo. Arq Bras Cardiol. 2006; 5:35360.

36. Farinatti PTV, Oliveira RB, Pinto VLM, Monteiro WD, Francischetti E. Programa Domiciliar de Exercícios: Efeitos de Curto Prazo sobre a Aptidão Física e Pressão Arterial de Indivíduos Hipertensos. Arq Bras Cardiol. 2005; 6:473-79.
37. Laterza MC, Rondon MUPB, Negrão CE. Efeito anti-hipertensivo do exercício. Rev Bras Hipertens.2007; 2:104-11.

38. Rabacow FM, Gomes MA, Marques P, Benedetti TB. Questionários de Medidas de Atividade Física em Idosos. Rev Bras Cineantropom Desempenho Hum. 2006; 4:99-106.

39. Florindo AA, Romero A, Peres SV, Silva MV, Slater B. Desenvolvimento e validade de um questionário de avaliação da atividade física para adolescentes. Rev Saúde Pública 2006; 5:802-9.

40. Glaner MF. Concordância de Questionários de Atividade Física com a Aptidão Cardiorrespiratória. Rev Bras Cineantropom Desempenho Hum 2007; 1:61-6.

41. Matos AC, Ladeia AM. Assessment of cardiovascular risk factors in rural community in the Brazilian state of Bahia. Arq Bras Cardiol. 2003; 3:297-302. 